\title{
Locating the Center of Mass of Various Simplified Car Designs for the Problem of Flipping Over an Incline
}

Sullivan Musgrove

University of South Florida, sullivanmusg@usf.edu

Advisors:

Arcadii Grinshpan, Mathematics and Statistics

Dmitri Voronine, Physics

Problem Suggested By: Dmitri Voronine

Field of Study for Problem Suggester: Physics

Follow this and additional works at: https://digitalcommons.usf.edu/ujmm

Part of the Mathematics Commons

UJMM is an open access journal, free to authors and readers, and relies on your support:

Donate Now

\section{Recommended Citation}

Musgrove, Sullivan (2020) "Locating the Center of Mass of Various Simplified Car Designs for the Problem of Flipping Over an Incline," Undergraduate Journal of Mathematical Modeling: One + Two: Vol. 11: Iss. 1, Article 6.

DOI: https://doi.org/10.5038/2326-3652.11.1.4925

Available at: https://digitalcommons.usf.edu/ujmm/vol11/iss $1 / 6$ 


\title{
Locating the Center of Mass of Various Simplified Car Designs for the Problem of Flipping Over an Incline
}

\author{
Abstract \\ The center of mass of a given system is referred to as a position that is the average of all of its \\ components. I am given two cases in which I need to find the center of mass for the problem of flipping \\ over an incline. To solve the problem given, I utilize many equations that are derived to find the center of \\ mass of both cases and then test each system when it is encountered with three different inclines \\ increasing by fifteen degrees increments. The tests prove that the probability that a system will flip on an \\ incline is due to many factors, the main of them being the height of the system, the area of support, as \\ well as the weight of the system. This method of solving is not the most time-efficient one to find the \\ center of mass and flipping probability; however, it does work and gives a viable solution.
}

\section{Keywords}

center of mass, center of gravity, centroid, moment, car design, flipping probability

Creative Commons License

(c) (i) (9)

This work is licensed under a Creative Commons Attribution-Noncommercial-Share Alike 4.0 License. 


\section{PROBLEM STATEMENT}

Calculate the center of mass of different car designs using simplified geometric shapes for the problem of flipping over an incline.

\section{Motivation}

The center of mass of a rigid object can be explained as the average position of all the components of a system, weighted according to their masses. There are many methods of finding centers of mass. For example, locating centers of mass with image processing is discussed in the recent UJMM article by Amina Gahramanova [1]. In the automotive industry, the center of mass of a given car is taken into account very vigorously in the design process as cars are encountered upon inclines, banks, and curves daily. For example, many semi-trucks and delivery trucks are driven up or down a loading dock that is set at an incline. If the center of mass of the given truck is too far in the back of the truck, the truck has a much higher chance of flipping from a lower incline. As well as a truck on an incline, many cars are encountered with curves and banks on public roads that have marginal inclines. These cars are sometimes traveling at high rates of speed and the center of mass of the car is an immense factor in the possibility of the given car to flip. In this paper, I will discuss two different cases of cars with simplified shapes and determine their center of mass to find out if the cars will flip on a given incline. This data and mathematical methods can be utilized in real-life problems when engineers are designing and manufacturing cars. 


\section{MATHEMATICAL DESCRIPTION AND SOLUTION APPROACH}

To gain a better understanding of finding the center of mass of simple geometric shapes it is useful to first look at Figure 1: there are two spheres of different masses attached by a thin bar of no mass.

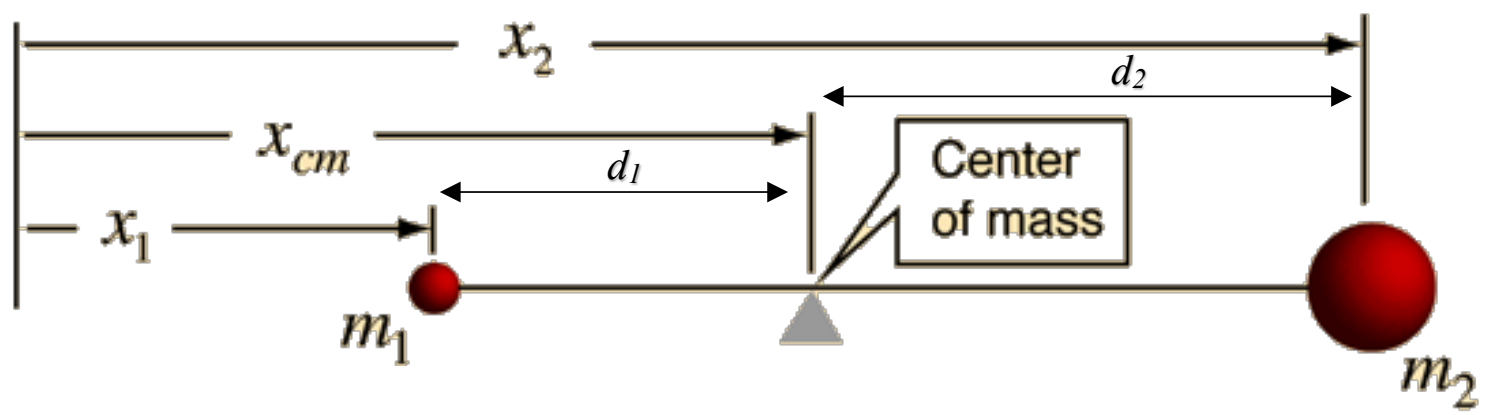

Figure 1: Two-Mass System with a representation of Center of Mass (source-hyperphysics.phyastr.gsu.edu)

To find the center of mass of this system so that the system is balanced on the point of the triangle one must use the following equation:

$$
\text { 1. } m_{1} d_{1}=m_{2} d_{2}
$$

With this equation known, one can assume that all of the coordinates lie on their respective points on the axis and the center of mass is at point $x_{\mathrm{cm}}$. Because I am working on a $2 \mathrm{D}$ plane with no masses given I can use the area of the objects in place of the masses. So, I will replace $m_{1}$ and $m_{2}$ with $a_{1}$ and $a_{2}$ :

$$
\text { 2. } a_{1} d_{1}=a_{2} d_{2}
$$

One can also see that $d_{1}=x_{c m}-x_{1}$ and $d_{2}=x_{2}-x_{c m}$. With these equations known, one can now substitute them into equation (1):

$$
\text { 3. } a_{1}\left(x_{c m}-x_{1}\right)=a_{2}\left(x_{2}-x_{c m}\right)
$$




$$
\begin{gathered}
\text { 4. } a_{1} x_{c m}+a_{2} x_{c m}=a_{1} x_{1}+a_{2} x_{2} \\
\text { 5. } x_{c m}=\frac{a_{1} x_{1}+a_{2} x_{2}}{a_{1}+a_{2}}
\end{gathered}
$$

Equation (5) is used for two masses respectively. When finding a centroid of many 2D masses and shapes one can use a similar method but one must find the centroid in the $x$ and $y$ directions. If I were given a graph with an origin and two $2 \mathrm{D}$ objects to find the center of mass, I would use the following equations.

$$
\begin{aligned}
& \text { 6. } x_{c m}=\frac{a_{1} x_{1}+a_{2} x_{2}}{a_{1}+a_{2}} \\
& \text { 7. } y_{c m}=\frac{a_{1} y_{1}+a_{2} y_{2}}{a_{1}+a_{2}}
\end{aligned}
$$

When encountering more than two 2D objects to find the center of mass one can just extend equations (5) and (6) and use $A=\sum_{i=1}^{n} a_{i}$ to get the following:

$$
\begin{aligned}
& \text { 8. } x_{c m}=\frac{\sum_{i=1}^{n} a_{i} x_{i}}{A} \\
& \text { 9. } y_{c m}=\frac{\sum_{i=1}^{n} a_{i} y_{i}}{A}
\end{aligned}
$$

One can use these equations as $x_{i}$ and $y_{i}$ are the distances from their respective axes to the midpoint of the shapes. When encountering shapes such as circles, squares, and rectangles, one can assume that they are symmetric, and in turn they have a mass at their center. In the illustration below, one can see how when dealing with symmetric shapes, the center of mass will be at the center of the shape shown by the red dot: 

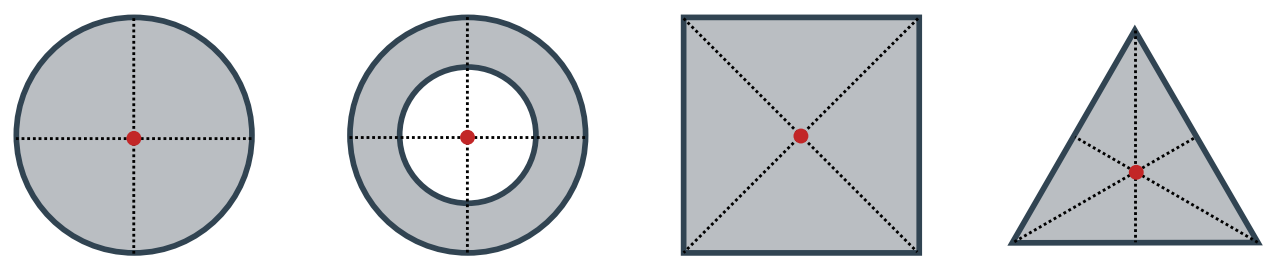

Figure 2: Center of Mass for some Simple Geometric Shapes (Red Dots) (sourcekhanacademy.org)

If one were to encounter an odd shape such as parabola that is non-symmetric one can use integration to then find the center of mass. For finding the center of mass of a given shape that is not symmetric one must first find the area of the given region using the equation (10) below.

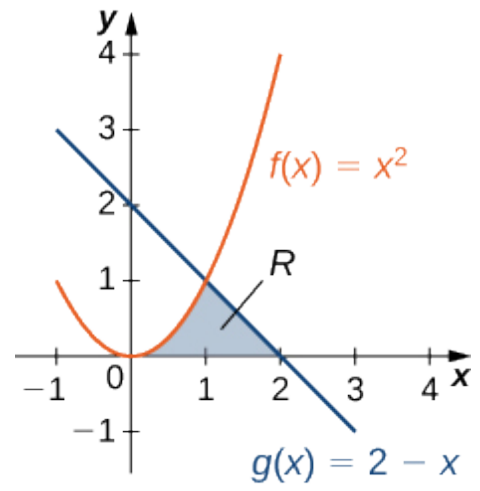

Figure 3: Calculating the area of the region (source- math.libretexts.org)

$$
\text { 10. } A=\int_{a}^{b}[f(x)-g(x)] d x, f(x) \geq g(x)
$$

After finding the area of the region one must find the moments. The moments are described as the measure of the region's tendency to rotate about the $x$ and $y$-axis. These moments are given by two equations, one for the $x$-direction, and one for the $y$-direction, similarly to the previous equations [2]:

$$
\begin{gathered}
\text { 11. } \bar{x}=\frac{1}{A} \int_{a}^{b} x[f(x)-g(x)] d x \\
\text { 12. } \bar{y}=\frac{1}{2 A} \int_{a}^{b}\left\{[f(x)]^{2}-[g(x)]^{2}\right\} d x
\end{gathered}
$$


With these equations, one can now locate the center of mass of a non-symmetric shape if the functions $f(x)$ and $g(x)$ as well as $a$ and $b$ are given $(f(x) \geq g(x), a \leq x \leq b)$. Now with the center of mass found of any given shape, one can use these points to determine the degree of an incline in which the shapes will topple. To calculate this, we are to know the center of mass of the object as well as the distance of the area of support and the degree of incline. The area of support of an object is given by the area that is in contact with the ground that is supporting the object. The larger this area of support the more stable the object is on an incline. The given reasoning states if the center of gravity falls outside of the area of support perpendicular to the ground, the object will overturn. However, if the center of gravity falls inside the area of support the given object will stay in place. This is why sports cars have a low center of gravity and a wide wheelbase (area of support) so that when taking hard turns and banks, the cars are less likely to overturn.

(a)

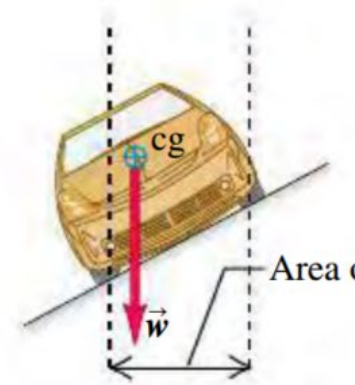

Center of gravity is over the area of support: car is in equilibrium. (b)

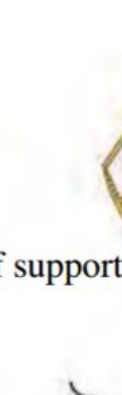

Center of gravity is outside the area of support: vehicle tips over.

(c)

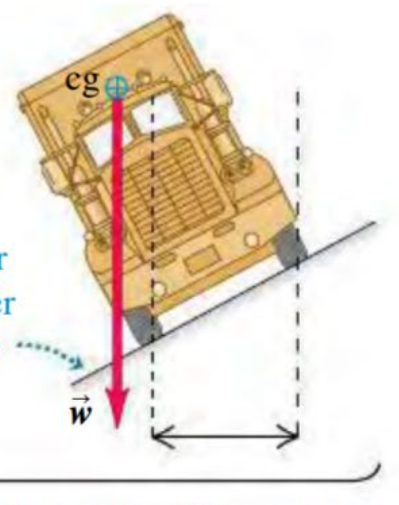

The higher the center of gravity, the smaller the incline needed to tip the vehicle over.

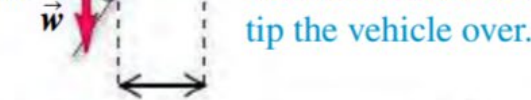

Figure 4: Different Car Designs and Area of Supports (source-etext.pearson.com) 
In Figure 4 one can see how the smaller car with the lower center of gravity will not overturn on the same incline as the truck. This is due to the car's wider wheelbase and a lower center of gravity.

\section{DISCUSSION}

For the problem of finding the center of mass of simplified cars, I am given two cases. The first case can be solved with the use of simplified geometric shapes such as a rectangle and two circles. With the rectangle acting as the body of the car and the two circles as the wheels of the car, this is the overall system viewed from a side profile. This is the shape of the car I am first given.

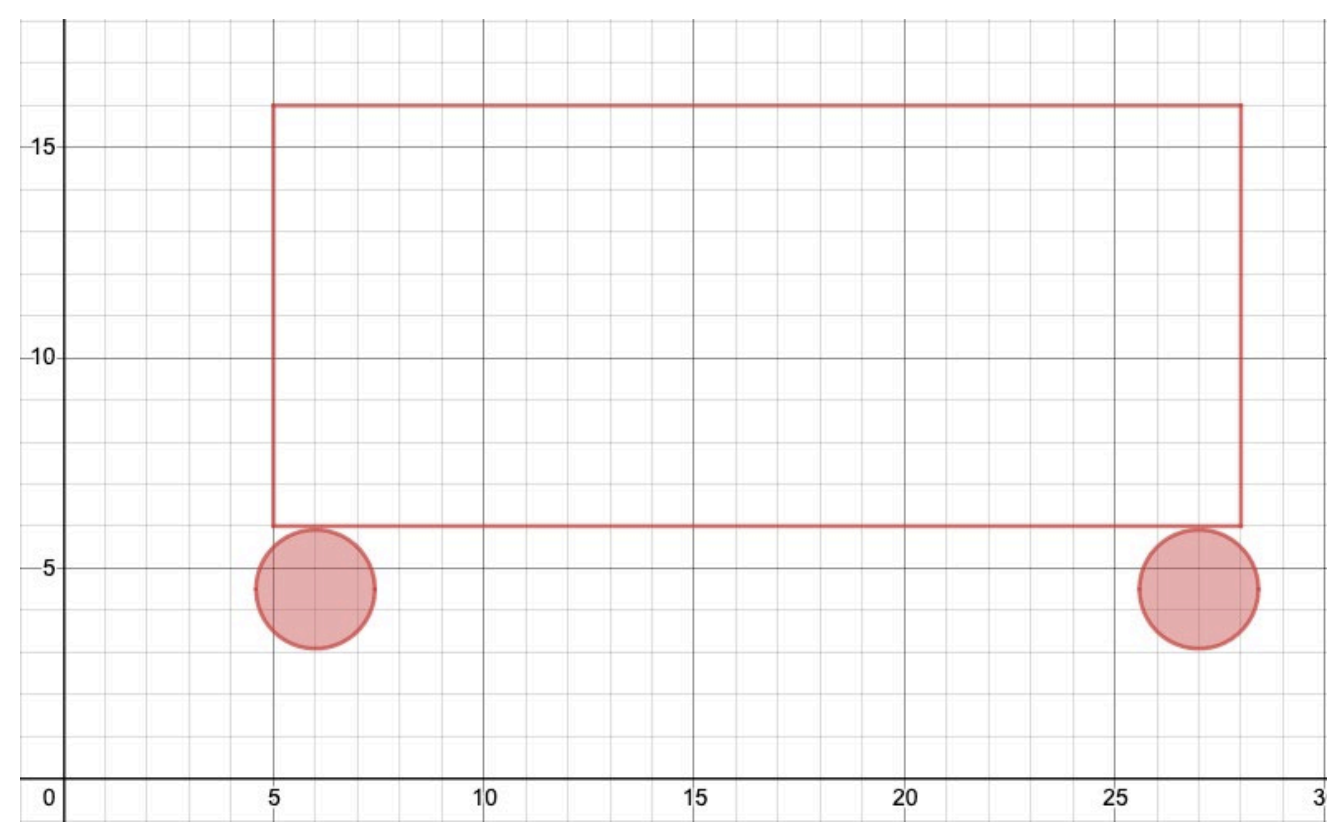

Figure 5: Case 1 car design made from (Created on desmos.com)

To find the center of mass of this car overall, I must first inspect the shapes of the drawing. I know that simple geometric shapes such as circles, squares, rectangles, and triangles have a center of mass that is at their true center because they are symmetric. So, I can find that the center of mass of the rectangle is at $(16.5,11)$ and the center of mass of the circles is at points 
$(6,4.5)$ and $(27,4.5)$ respectively. With this information, I can find the center of mass of the system as a whole to be $(16.5,10.6236)$ using the equations $(8)$ and (9) (see Appendix).

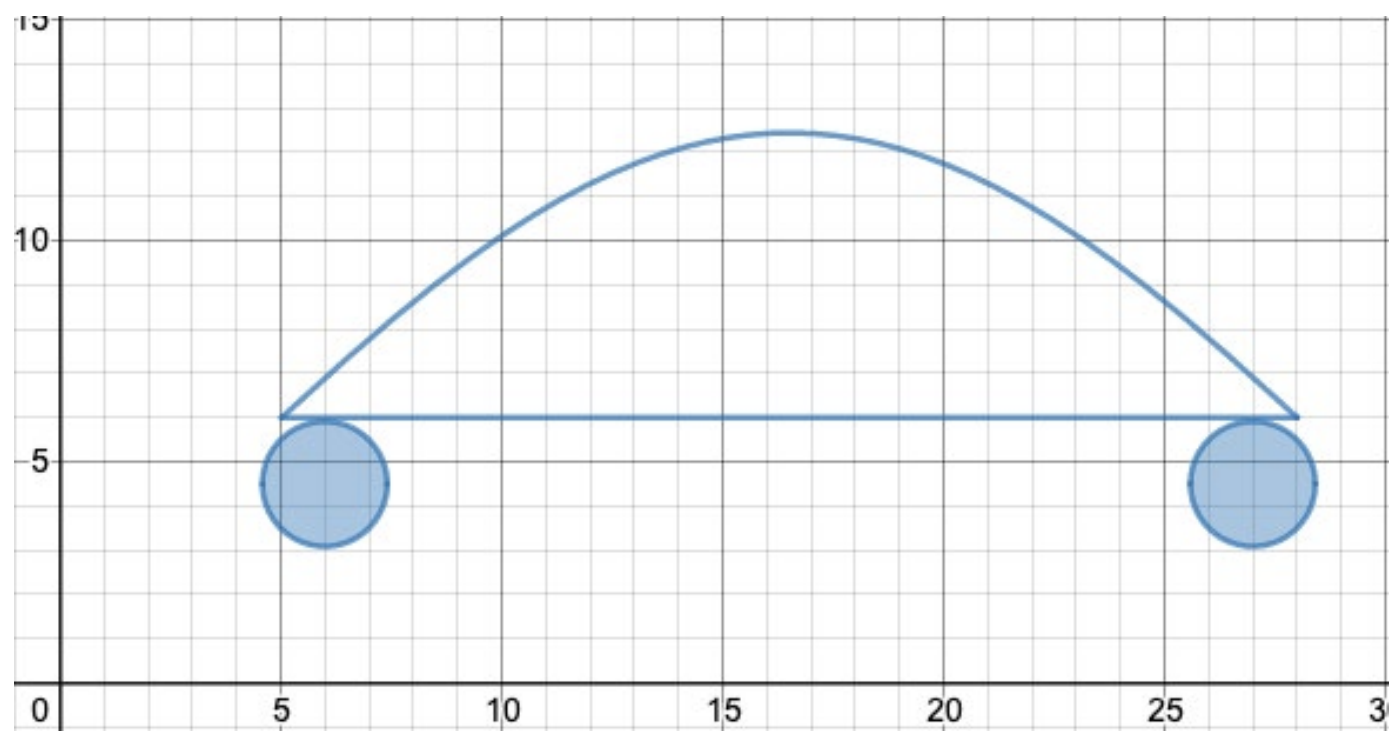

Figure 6: Case 2 car design made from (Created on desmos.com)

Finding the center of mass of the car above (Figure 6) is much harder than the previous case as I have to find the area under the "parabola" as well as the center of mass of the shape created by the lines $y=6$ and $y=5+7.44 \sin \left(\frac{1}{8} x+5.79\right)$. To find the area between the two curves I use equation (10) (see Appendix):

$$
\begin{gathered}
\text { 13. } A=\int_{5}^{28}\left[5+7.44 \sin \left(\frac{1}{8} x+5.79\right)-6\right] d x \\
=\int_{5}^{28} 7.44 \sin \left(\frac{1}{8} x+5.79\right) d x-\int_{5}^{28} d x \\
=117.98352-23=94.983889
\end{gathered}
$$

So, the area under the given lines is seen to be $94.983889 \mathrm{~m}^{2}$. With the area found I can now find moments at the given $x$ and $y$ points using equations (11) and (12) (see Appendix).

$$
14 . \bar{x}=\frac{1}{94.983889} \int_{5}^{28} x\left[5+7.44 \sin \left(\frac{1}{8} x+5.79\right)-6\right] d x
$$




$$
\begin{gathered}
=\frac{1}{94.983889}\left\{-\int_{5}^{28} x d x+\int_{5}^{28} 7.44 x \sin \left(\frac{1}{8} x+5.79\right) d x\right\} \\
=\frac{1568.36308}{94.983889}=16.51188 \\
15 . \bar{y}=\frac{1}{2 \cdot 94.983889} \int_{5}^{28}\left[\left(5+7.44 \sin \left(\frac{1}{8} x+5.79\right)\right)^{2}-6^{2}\right] d x \\
=\frac{1}{189.967778}\left\{\int_{5}^{28} 55.3536 \sin ^{2}\left(\frac{x}{8}+5.79\right) d x+\int_{5}^{28} 74.4 \sin \left(\frac{x}{8}+5.79\right) d x-\int_{5}^{28} 11 d x\right\} \\
=\frac{694.897+1179.84-253}{189.967778}=8.5369
\end{gathered}
$$

Through using equations (10), (11) and (12) I am able to find the area under the curve which in turn allows me to calculate the center of mass at $(16.51188,8.5369)$. I then use these moments to find the center of mass of the system as a whole. I have already found the area and center of mass of the two circles in case 1, so I can reuse the numbers found through calculations. Using equations (8) and (9) I plug in our centers of mass, as well as areas of each shape created to get a center of mass of the system to be at point $(16.5103,8.0139)$. With the center of masses of each system known I can now solve for their problem of flipping over an incline. For this problem, I am going to test each car design on the incline of $30^{\circ}, 45^{\circ}$, and $60^{\circ}$. The figures below will contain the system on an incline with the arrows coming from each wheel representing the area of support of the car, while the arrow coming from the center of mass will be representing the point where gravity will be acting on the system. From the mathematical approach I have learned that if the center of mass falls outside the area of supports then the car will flip. 

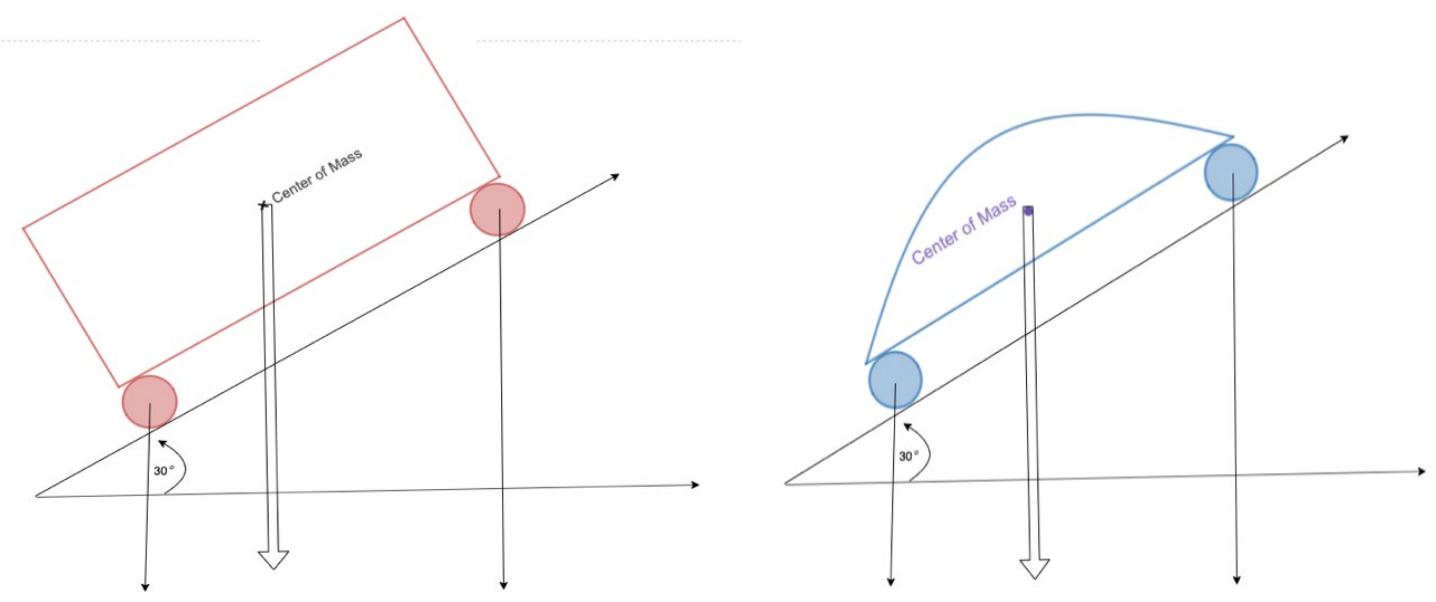

Figure 7: Systems on a $30^{\circ}$ incline.
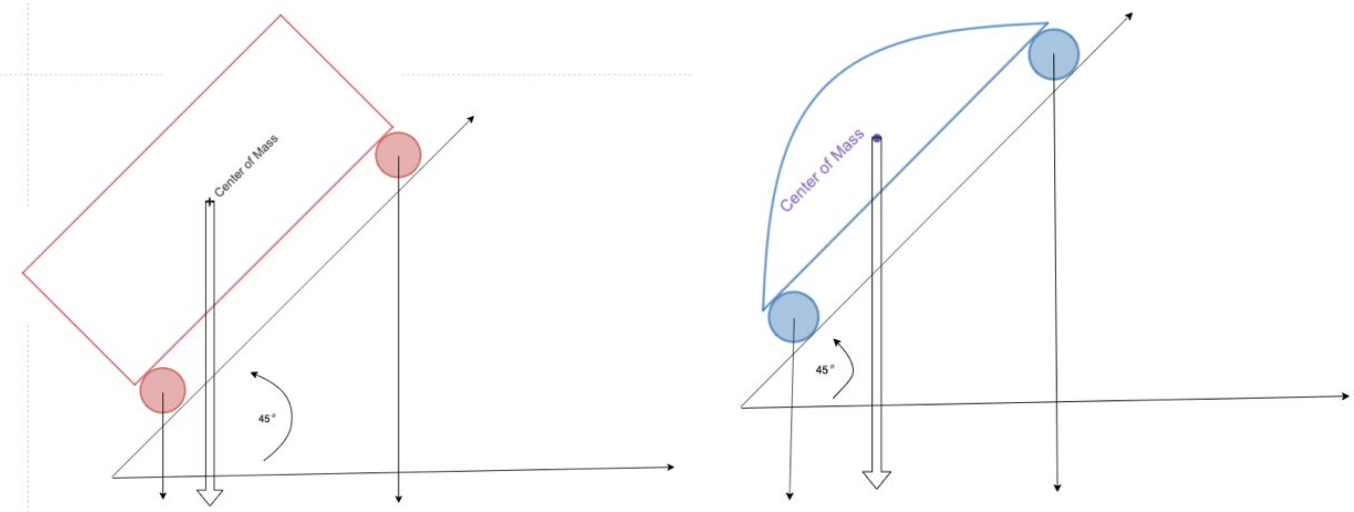

Figure 8: Systems on a $45^{\circ}$ incline.
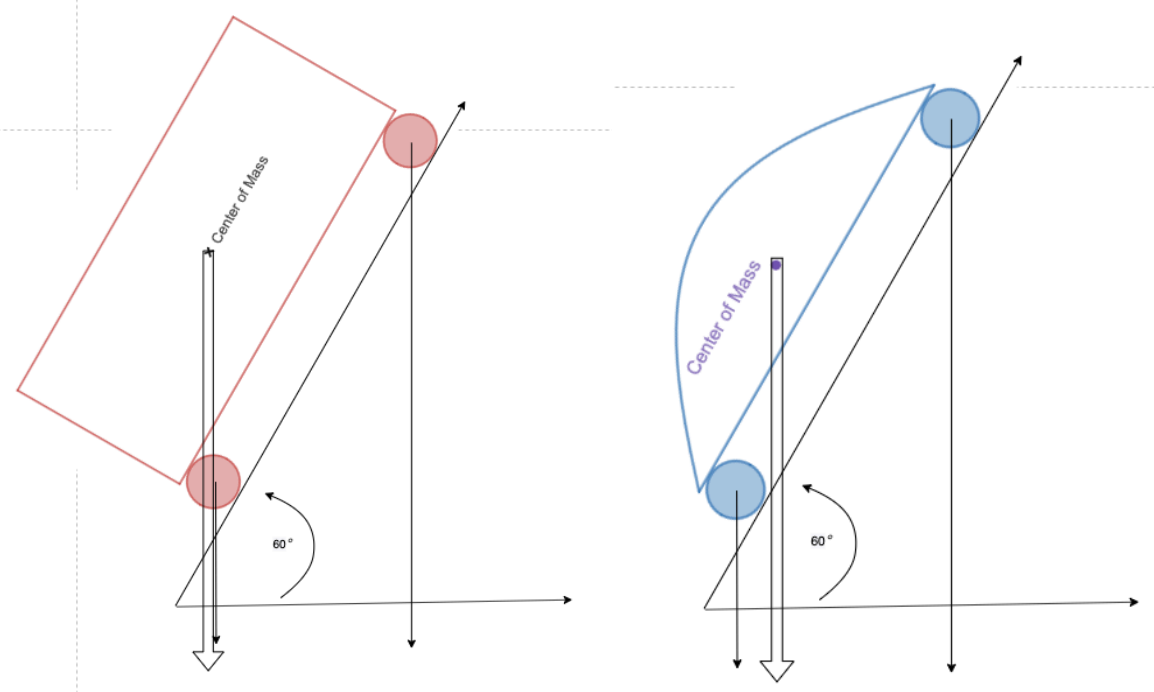

Figure 9: Systems on a $60^{\circ}$ incline. 
It is seen that at the $30^{\circ}$ and $45^{\circ}$ inclines, the systems would not flip as the center of mass fell inside the area of support. While on the $60^{\circ}$ incline, the first system that was taller would have flipped over while the second system would have not. This is due to the center of mass of the first system being higher up because of the square shape and having more mass higher up in the system.

\section{CONCLUSION AND RECOMMENDATIONS}

To solve the problem of flipping over an incline for two given cases I was to find the center of mass of cars utilizing simplified geometric shapes. To do this I derived many equations that were needed. Using simple algebra and integration techniques I was able to calculate the center of mass of both cases, and then use them to determine if the car would flip over a $30^{\circ}, 45^{\circ}$, and a $60^{\circ}$ incline from the side plane. I was able to determine that when comparing two cars with the same wheelbase and axle length the taller car was more likely to flip on a lower incline. The height, weight, and wheelbase are all large factors that play into the tipping of a car. For example, when comparing a Jeep Wrangler with a small sedan such as a Hyundai Sonata, the Jeep is much more likely to flip as the wheelbase is much shorter than that of the Hyundai's. With this known other students can use this process for real-life situations when encountering cars. One could even use this process when dealing with a small toy car such as someone who races $\mathrm{RC}$ cars. In the future, I would like to also follow the same procedure for the front view of a car as well as the side. This will give more information on the true build quality of the car. This will also give more information to readers on how the design of a car is taken into account overall as one must look at the whole car rather than just one side. 
M usgrove: Locating the Center of Mass of V arious Simplified Car Designs for

NOMENCLATURE

\begin{tabular}{|c|c|c|}
\hline Symbol & Description & Unit \\
\hline$m$ & Mass & $\mathrm{m}^{2}$ \\
\hline$A$ & Total Area & $\mathrm{m}^{2}$ \\
\hline$a$ & Area & $\mathrm{m}$ \\
\hline $\bar{x}$ & Moment in $x$-direction & $\mathrm{m}$ \\
\hline $\bar{y}$ & Moment in $y$-direction & $\mathrm{m}$ \\
\hline$d$ & Distance & \multicolumn{2}{|c|}{} \\
\hline
\end{tabular}




\section{REFERENCES}

1. Amina Gahramanova (2019). Locating Centers of Mass with Image Processing, Undergraduate Journal of Mathematical Modeling: One + Two: Vol. 10: Iss. 1, Article 1.

2. James Stewart (2012). Essential Calculus: Early Transcendentals. Mason, Ohio: Cengage Learning.

3. Hugh D. Young, Roger A. Freedman (2016). University Physics with Modern Physics. 14th ed., Pearson Higher Education.

\section{Websites}

1. Nave, R. Center of Mass, http://hyperphysics.phy-astr.gsu.edu/hbase/cm.html.

2. "What Is Center of Mass?" Khan Academy, Khan Academy, https://www.khanacademy.org/science/physics/linear-momentum/center-of-mass/a/what-iscenter-of-mass.

3. Libretexts. “6.1: Areas between Curves.” Mathematics LibreTexts, Libretexts, 21 Oct. 2019, https://math.libretexts.org/Bookshelves/Calculus/Book:_Calculus_(OpenStax)/6:_Applications_o f_Integration/6.1:_Areas_between_Curves.

4.“Explore Math with Desmos.” Desmos.com, https://www.desmos.com/. 


\section{APPENDICES}

Appendix 1: Calculations

\section{Case 1}

Area of the rectangle: $230 \mathrm{~m}^{2}$

Area of each disk (radius is 1.5 ): $2.25 \pi \mathrm{m}^{2}$

$x_{c m}=\frac{16.5 \times 230+6 \times 2.25 \times \pi+27 \times 2.25 \times \pi}{230+2 \times 2.25 \pi}=16.5$

$y_{c m}=\frac{11 \times 230+4.5 \times 2.25 \times \pi+4.5 \times 2.25 \times \pi}{230+2 \pi \times 2.25}=10.6236 \ldots$

\section{Case 2}

Finding the area

$$
\begin{aligned}
& A=\int_{5}^{28}\left[5+7.44 \sin \left(\frac{1}{8} x+5.79\right)-6\right] d x \\
& =\int_{5}^{28} 7.44 \sin \left(\frac{1}{8} x+5.79\right) d x-\int_{5}^{28} d x
\end{aligned}
$$

We obtain:

$$
\begin{aligned}
& \int_{5}^{28} 7.44 \sin \left(\frac{1}{8} x+5.79\right) d x \\
& =7.44 \cdot 8 \cdot \int_{6.415}^{9.29} \sin (u) d u=59.52[-\cos (u)]_{6.415}^{9.29}=117.983889 \ldots \\
& \int_{5}^{28} d x=23 \\
& A=117.983889-23=94.983889
\end{aligned}
$$

Finding $x$-moment:

$$
\bar{x}=\frac{1}{94.983889} \int_{5}^{28} x\left[5+7.44 \sin \left(\frac{1}{8} x+5.79\right)-6\right] d x
$$

We have:

$$
\begin{aligned}
& \int_{5}^{28} x\left[-1+7.44 \sin \left(\frac{1}{8} x+5.79\right)\right] d x \\
& =-\int_{5}^{28} x d x+\int_{5}^{28} 7.44 x \sin \left(\frac{1}{8} x+5.79\right) d x \\
& =\left[-\frac{x^{2}}{2}-59.52 x \cos \left(\frac{1}{8} x+5.79\right)+476.16 \sin \left(\frac{1}{8} x+5.79\right)\right]_{5}^{28}=1568.36308
\end{aligned}
$$


$\bar{x}=\frac{1568.36308}{94.983889}=16.51188$

Finding $y$-moment:

$$
\begin{gathered}
\bar{y}=\frac{1}{2 \cdot 94.983889} \int_{5}^{28}\left[\left(5+7.44 \sin \left(\frac{1}{8} x+5.79\right)\right)^{2}-6^{2}\right] d x \\
=\frac{1}{189.967778}\left\{\int_{5}^{28} 55.3536 \sin ^{2}\left(\frac{x}{8}+5.79\right) d x+\int_{5}^{28} 74.4 \sin \left(\frac{x}{8}+5.79\right) d x-\int_{5}^{28} 11 d x\right\} ;
\end{gathered}
$$

We obtain:

$55.3536 \cdot \int_{5}^{28} \sin ^{2}\left(\frac{x}{8}+5.79\right) d x=55.3536 \cdot 8 \int_{6.415}^{9.29} \sin ^{2}(u) d u$

$=55.3536 \cdot 8 \cdot \frac{\int_{6.415}^{9.29}((1-\cos (2 u))}{2} d u$

$=55.3536 \cdot 4[2.875-(\sin (18.58)-\sin (12.83)) / 2]$

$=110.7072 \cdot 3.13844=694.897$;

$74.4 \cdot \int_{5}^{28} \sin \left(\frac{x}{8}+5.79\right) d x=74.4 \cdot 8 \int_{6.415}^{9.29} \sin (u) d u$

$=74.4 \cdot 8[-\cos (u)]_{6.415}^{9.29}=595.2 \cdot 1.982256=1179.83889$;

$\int_{5}^{28} 11 d x=253$

$$
\bar{y}=\frac{694.897+1179.84-253}{2 \cdot 94.983889}=8.53690566
$$

